\title{
Neuroprotective effects of lithium on a chronic MPTP mouse model of Parkinson's disease via regulation of $\alpha$-synuclein methylation
}

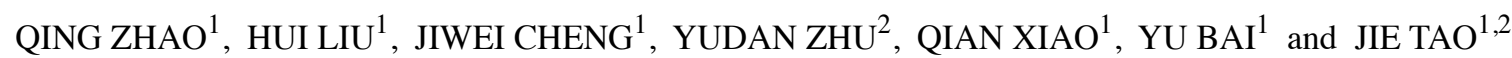 \\ ${ }^{1}$ Department of Neurology; ${ }^{2}$ Central Laboratory, Putuo Hospital, \\ Shanghai University of Traditional Chinese Medicine, Shanghai 200062, P.R. China
}

Received September 18, 2018; Accepted April 2, 2019

DOI: $10.3892 / \mathrm{mmr} .2019 .10152$

\begin{abstract}
The pathological process of Parkinson's disease (PD) is closely associated with the death of nigral neurons, for which an effective treatment has yet to be found. Lithium, one of the most widely certified anticonvulsant and mood-stabilizing agents, exhibits evident neuroprotective effects in the treatment of epilepsy and bipolar disorder. In the present study, the neuroprotective mechanisms by which lithium acts on a chronic 1-methyl-4-phenyl-1,2,3,6-tetrahydropyridine (MPTP) mouse model of PD were investigated by employing animal behavioral tests, immunohistochemistry, RT-PCR, and western blotting. The results revealed that, in open field tests, lithium treatment counteracted the reduction in movement distance as well as activity time induced by MPTP administration. The compound could also prolong the drop time of MPTP-treated mice in rotarod tests. Moreover, lithium treatment corrected the loss of nigral neurons, the increase of $\alpha$-synuclein (SNCA) in substantia nigra as well as in the striatum of MPTP-treated mice, and decreased the methylation of SNCA intron 1 in DNA from the same regions. Furthermore, marked changes were observed in the expression of miRNAs including miR-148a, a potential inhibitor of DNMT1, in the MPTP-treated mice. These results suggested that the early application of lithium was important for alleviating the behavioral deficits experienced in the PD model, and that the neuroprotective action of lithium was achieved through a lithium-triggered miRNA regulation mechanism. Essentially, our findings indicated that
\end{abstract}

Correspondence to: Dr Qing Zhao, Department of Neurology, Putuo Hospital, Shanghai University of Traditional Chinese Medicine, 164 Lanxi Road, Putuo, Shanghai 200062, P.R. China E-mail: qingzhao2010@hotmail.com

Dr Jie Tao, Central Laboratory, Putuo Hospital, Shanghai University of Traditional Chinese Medicine, 164 Lanxi Road, Putuo, Shanghai 200062, P.R. China

E-mail: jietao_putuo@foxmail.com

Key words: Parkinson's disease, DNA methylation, lithium, $\alpha$-synuclein, nigral neuron lithium may be beneficial in the prevention and treatment of PD through the regulation of $\alpha$-synuclein methylation.

\section{Introduction}

Parkinson's disease (PD) is the second most common neurodegenerative disorder worldwide and affects $1 \%$ of the population over the age of 60 (1). This neurodegenerative disorder is characterized by the progressive loss of substantia nigra dopaminergic neurons as well as striatal projections, which causes the typical symptomatology, including bradykinesia, tremor, muscle rigidity, and postural instability (1). However, only $5-10 \%$ of PD cases are considered to involve genetic factors (1). Although recent studies have found that several factors play a key role in the neuronal pathogenesis of PD (2), the etiology of PD remains elusive. It is now considered that $\alpha$-synuclein (SNCA), a major component of Lewy bodies, is one of the morphological markers of PD. Several site mutations in SNCA have been identified as the cause for early onset PD in a dominant mode of inheritance (3). Moreover, methylation of intron 1 of human SNCA results in reduction of gene expression in the brains of PD patients, suggesting that methylation of SNCA is correlative with PD pathogenesis (3). Despite the lack of any differences in the postmortem analysis of regional specific methylation at the anterior cingulate or putamen of the brains of PD patients and healthy individuals, methylation in the substantia nigra of PD patients was significantly and specifically decreased (4). Furthermore, single $\mathrm{CpG}$ analysis revealed a fluctuation in the methylation levels of various brain regions and LBD stages, which is suggestive of a potential role for DNA methylation of $\alpha$-synuclein in the occurrence of PD (5).

One of the first-line antiepileptic drugs (AEDs) is lithium, a drug that is also used in the treatment of bipolar disorder (6). Similar to other anticonvulsants, lithium inhibits some of the functions of sodium and calcium channels (6). Despite the need for deeper investigation into its in vivo target, lithium treatment could induce a significant change in the activity of histone deacetylase (HDAC) as well as glycogen synthase kinase (GSK-3). Further studies have revealed that the inhibition of GSK-3 $\beta$ activity induced by lithium mimics resulted in a reduction of DNA methylation in neural stem cells (7). 
Similar to valproic acid (VPA), lithium was recently demonstrated to be an effective regulator for the expression of several PD-related miRNAs (6). In neuroblastoma cells, lithium treatment attenuated the apoptosis induced by rotenone, an inhibitor of mitochondrial complex 1 that induces PD-like neurodegeneration in vivo (8). It has also been suggested that by enhancing the autophagic pathway that is associated with the degradation of aberrant accumulated $\alpha$-synuclein protein in PD, lithium could, act as a neuroprotective agent in rotenone-induced SH-SY5Y cells as well as in MPTP-lesioned mice $(6,8-10)$. Other studies reported that lithium could increase the expression of neurotrophins, which are involved in neural survival as well as plasticity, for example the nerve growth factors (NGF), brain-derived neurotrophic factor (BDNF), and the glial cell line-derived neurotrophic factor (GDNF) $(11,12)$.

Herein, in order to detect the effects of lithium on the neurodegenerative symptoms using behavioral tests, a commonly used PD-like mouse model was employed, and the animals were fed chow with lithium carbonate for 5 weeks. Biochemical assays were conducted to explore the molecular events associated with PD pathogenesis and further elucidate the relationship between lithium and the development and progression of this disease.

\section{Materials and methods}

Animals. A total of 80 male C57BL/6 mice (7-8 weeks, $25 \mathrm{~g}$ ) were obtained from the Shanghai Experimental Animal Center of Chinese Academy of Sciences (Shanghai, China). Each individual cage contained 4 mice, with free access to water and food. All of the animal rooms were maintained at a temperature of $21-23^{\circ} \mathrm{C}$ and humidity of $40-60 \%$, with a 12-h light-dark cycle. To develop the MPTP PD model, male C57BL/6J mice were injected intraperitoneally (i.p.) with 1-methyl-4-phenyl-1,2,3,6-tetrahydrapyridine (MPTP) at $20 \mathrm{mg} / \mathrm{kg}$ (body weight; Sigma-Aldrich; Merck KGaA, Darmstadt, Germany) twice a week, plus $250 \mathrm{mg} / \mathrm{kg}$ probenecid injected $0.5 \mathrm{~h}$ before. Administration of the treatment was carried out for 5 weeks with feeding of $0.2 \%$ lithium carbonate (Sigma-Aldrich; Merck KGaA) by chow. On the 36th day, the mice were subjected to behavioral tests. Upon completion, mice were anesthetized with $250 \mathrm{mg} / \mathrm{kg}$ avertin intraperitoneally and perfused transcardially with sterile saline. Brains were quickly harvested, and one hemibrain was immediately frozen in liquid nitrogen for biochemical studies while the other hemibrain was post-fixed in $4 \%$ paraformaldehyde for immunohistochemical stains. All animal procedures complied with the current ethical considerations of the Shanghai University of Traditional Chinese Medicine's Animal Ethics Committee, which is in accordance with the National Research Council criteria. All animal experiments were reviewed and approved by the Institutional Animal Care and Use Committee (IACUC) of Shanghai University of Traditional Chinese Medicine and were performed in accordance with the relevant guidelines and regulations as well as approved by the guidelines on ethical standards for investigation of PD in conscious animals. Animals were sedated or anesthetized using carbon dioxide prior to cervical dislocation. Cervical dislocation to euthanize mice was performed by trained research personnel after the approval of the IACUC of Shanghai University of Traditional Chinese Medicine's Animal Ethics Committee and the method was performed in accordance with the American Veterinary Medical Association (AVMA) Guidelines for the Euthanasia of Animals (2013 Edition).

\section{Behavioral tests}

Open-field test. The open field activity was assessed in a $27 \times 27 \times 38 \mathrm{~cm}$ chamber with 50 lux (lx) illumination. Fifteen minutes of free movement was tracked by TruScan Open Field version 2.04 software 2.04 (Coulbourn Instruments, Holliston, MA, USA). Total movement times and distances were scored to assess locomotor activity while a percentage of movement time in the margin area (the area within $8.76 \mathrm{~cm}$ from the chamber wall) was calculated for scrutinizing the autonomous activity (13).

Rotarod performance test. Rotarod training was performed concurrently for 10 min during 5 consecutive days: On the first day, the mice were placed on the rotating rod at $1.5 \mathrm{x} \mathrm{g}$ for $5 \mathrm{~min}$. Every $30 \mathrm{sec}$, the rod speed was increased by $0.4 \mathrm{x} \mathrm{g}$ up to $5.5 \mathrm{x} \mathrm{g}$, and then maintained at this speed for $1 \mathrm{~min}$. On the second day, a $1.5 \mathrm{x}$ g speed was used for $1.5 \mathrm{~min}$. The rod speed was increased by $0.4 \mathrm{x}$ g every $30 \mathrm{sec}$ until it reached $7.3 \mathrm{x} \mathrm{g}$, where it was maintained for $10 \mathrm{~min}$. On the third, fourth, and fifth days, the latencies of falling off the rod with a linear increase in rod speed from $1.5 \mathrm{x} \mathrm{g}$ up to $15 \mathrm{x} \mathrm{g}$ for $5 \mathrm{~min}$ were measured 3 times and averaged. The actual test protocol was the same for the last 3 days of the training protocol.

Bisulfite conversion and methylation-specific-PCR (MSP). For the bisulfite modification, the EZ DNA Methylation-Gold ${ }^{\mathrm{TM}}$ Kit (Zymo Research Corp., Irvine, CA, USA) was used. In this technique, unmethylated cytosines are converted to uracil while methylated cytosines remain unchanged in bisulfite-treated DNA. Thus, the methylation status of the DNA sample can be analyzed by PCR amplification, called 'methylation specific-PCR.' DNA $(\sim 1 \mu \mathrm{g})$ was used for bisulfite conversion, and then MSP was carried out using specific primers for both bisulfite converted methylated and unmethylated DNA samples in a total $25 \mu \mathrm{l}$ mix containing 1X PCR buffer, $15 \mathrm{mM} \mathrm{MgCl}_{2}, 200 \mu \mathrm{M}$ of each dNTP, $0.4 \mu \mathrm{M}$ of each primer, $<1 \mu \mathrm{g}$ template DNA, and $2.5 \mathrm{U}$ HotStarTaq DNA Polymerase (Qiagen, Inc., Valencia, CA, USA). After the initial denaturation step at $94^{\circ} \mathrm{C}$ for $15 \mathrm{~min}, 35$ cycles were performed for the regions of SNCA using the following conditions: denaturation at $94^{\circ} \mathrm{C}$ for $1 \mathrm{~min}$, at annealing temperature for each PCR bisulfide conversion-specific primer pair for $1 \mathrm{~min}$, extension at $72^{\circ} \mathrm{C}$ for $1 \mathrm{~min}$, and then final extension at $72^{\circ} \mathrm{C}$ for $10 \mathrm{~min}$. Bisulfite-treated DNA was amplified using primers SNCA-PromF, 5'-AAAATTTTGAAGATA TTTGAATTAAAG-3' and SNCA-PromR, 5'-CTAATCCTC CTCCTTCTCCTTCTC-3'; SNCA-IntF, 5'-GGAGTTTAA GGAAAGAGATTTGATT-3' and SNCA-IntR, 5'-CAAACA ACAAACCCAAATATAATAA-3', specifically designed for the bisulfite-treated DNA. The PCR products SNCA (-926/-483; intron 1) were cloned into pMD 18-T Vector (Takara Biotechnology Co., Ltd., Dalian, China) following the manufacturer's instructions. Plasmid DNA was isolated from at least 10 clones per region (Wizard Plus SV Minipreps; Promega Corporation, Madison, WI, USA), sequenced using vector-specific primers and the Big Dye Terminator v1.1 
Cycle Sequencing Kit (Applied Biosystems; Thermo Fisher Scientific, Inc., Waltham, MA, USA), and analyzed on an ABI PRISM 310 Genetic Analyzer (Applied Biosystems; Thermo Fisher Scientific, Inc.). Quality control for DNA methylation was performed using BiQ (software tool for DNA methylation analysis; http://biq-analyzer.bioinf.mpi-inf.mpg.de/).

Reverse transcription-quantitative PCR (RT-qPCR). Total RNA, including mRNA and miRNA, was isolated from samples using an RNeasy Mini Kit (Qiagen, Inc.). Next, cDNA was synthesized using a PrimeScript ${ }^{\mathrm{TM}} \mathrm{RT}$ reagent kit (Takara Biotechnology Co., Ltd.) and subsequently used as templates for qPCR. The primers were designed using Primer 5.0 (Premier Biosoft International, Palo Alto, CA, USA). Primers of real-time PCR were SNCA F, 5'-GGACCAGTT GGGCAAGAATG-3' and R, 5'-GGGCACATTGGAACT GAGCAC-3'; GAPDH F, 5'-CGGAGTCAACGGATTTGG TC-3' and R, 5'-TTCTCCATGGTGGTGAAGAC-3'; miR-148a F, 5'-TCAGTGCACTACAGAACTTTGT-3' and R, 5'-GCT GTCAACGATACGCTACG-3'; U6 F, 5'-CTTCGGCAGCAC ATATAC-3' and R, 5'-GAACGCTTCACGAATTTGC-3'. qPCR was performed in triplicate with SYBR-Green (Takara Biotechnology Co., Ltd.) on an Applied Biosystems 7500 Fast Real-Time PCR System (Applied Biosystems; Thermo Fisher Scientific, Inc.). The reaction parameters were set as follows: $95^{\circ} \mathrm{C}$ for $5 \mathrm{~min}$, followed by 40 cycles of $10 \mathrm{sec}$ for $95^{\circ} \mathrm{C}$ and $1 \mathrm{~min}$ for $60^{\circ} \mathrm{C}$. For miRNA RT-qPCR, $1 \mathrm{mg}$ of isolated RNA was reverse-transcribed with stem-loop primers from a BioTNT miRNA qPCR Detection Primer Set (BioTNT Biotechnologies Co, Ltd., Shanghai, China). All reactions were repeated in triplicate. The relative mRNA and miRNA expression levels were separately analyzed using the $2^{-\Delta \Delta C q}$ method (14) with GADPH and U6 as the endogenous controls.

miRNA expression microarrays. Total RNA (including miRNAs) was isolated from the brain tissue of the substantia nigra using TRIzol ${ }^{\circledR}$ (Thermo Fisher Scientific, Inc.) with slight modification. Polyacryl carrier was added to improve RNA recovery. The global miRNA expression profiles were obtained using the Agilent SurePrint Mouse miRNA Microarrays (Agilent Technologies, Inc., Santa Clara, CA, USA) containing 1,881 mouse miRNAs based on Sanger miRBase release 12.0. RNA samples were processed, labeled, and hybridized onto the microarrays according to the manufacturer's protocols. Microarray slides were then scanned using the G3 High Resolution Scanner, and microarray data were extracted by Feature Extraction software version 10.7.3.1 (Agilent Technologies, Inc.).

Immunohistochemistry. Animals were anaesthetized with ether and perfused through the heart with $4 \%$ paraformaldehyde in $0.1 \mathrm{mM}$ phosphate buffer $\mathrm{pH}$ 7.4. Brains were removed, post-fixed overnight in the same fixative, and then washed in buffered $18 \%$ sucrose until they sunk. Sections were cut with a freezing microtome at 30- $\mu \mathrm{m}$ thickness, and then permeabilized for 20 min with phosphate-buffered saline (PBS) containing $0.1 \%$ Triton $\mathrm{X}-100$, before a 20 -min incubation in methanol containing $0.3 \% \mathrm{H}_{2} \mathrm{O}_{2}$ to quench endogenous peroxidase activity. Subsequently, the sections were incubated for $30 \mathrm{~min}$ in PBS containing 2\% normal goat serum to block non-specific binding sites. For immunohistochemical localization of tyrosine hydroxylase $(\mathrm{TH})$, the $\mathrm{TH}$ antibody was used at a dilution of 1:1,000 (cat. no. sc-136100, Santa Cruz Biotechnology, Inc., Dallas, TX, USA). Antibody exposure was performed overnight at $4^{\circ} \mathrm{C}$, followed by a 90 -min incubation at room temperature with a horseradish peroxidase-conjugated goat anti-mouse immunoglobulin G (IgG) secondary antibody (cat. no. A0216, Beyotime Institute of Biotechnology, Haimen, China). The immunoreaction was visualized using DAB Horseradish Peroxidase Color Development Kit (Beyotime Institute of Biotechnology). Digital images were captured using a Leica DM2000 Microscopic imaging system, and TH-positive neuronal counts and the integrated optical density value (IOD) of $\alpha$-synuclein-positive fibers were estimated within the substantia nigra by ImageJ software (NIH) as previously described (15).

Western blotting. The substantia nigra of wild-type, MPTP-treated, and lithium-treated mice $(n=3)$, were dissected, pooled, and homogenized in lysis buffer (Beyotime Institute of Biotechnology) on ice plus 1:100 volume of phenylmethylsulfonyl fluoride (PMSF), before final centrifugation at $14,000 \mathrm{x} \mathrm{g}$ for $5 \mathrm{~min}$ to remove debris. The BCA kit (Beyotime Institute of Biotechnology) was used to assess the protein concentrations. After heating at $100^{\circ} \mathrm{C}$ with loading buffer for $4 \mathrm{~min}$, the proteins $(30 \mu \mathrm{g})$ were resolved by SDS-PAGE (12\% for a-synuclein, 6\% for DNMT1) and transferred to nitrocellulose membranes (Amersham; GE Healthcare, Chicago, IL, USA) at $200 \mathrm{~mA}$ for $40 \mathrm{~min}$. Tris-buffered saline and Tween-20 (TBST) containing 5\% skimmed milk powder was used to block the membranes at room temperature for $1 \mathrm{~h}$, and then membranes were washed with TBST prior to incubation with antibodies against $\alpha$-synuclein (ab27766, 1:2,000; Abcam, Cambridge, MA, USA) and DNMT1 (ab19905, 1:2,000; Abcam) overnight at $4^{\circ} \mathrm{C}$. After washing thrice with TBST, the membranes were incubated with horseradish peroxidase conjugated anti-rabbit (cat. no. A0208) or anti-mouse IgG (cat. no. A0216, Beyotime Institute of Biotechnology) at a dilution of 1:2,000 at room temperature for $45 \mathrm{~min}$. After washing for three further times with TBST, the proteins were visualized using an enhanced chemiluminescence kit (EMD Millipore, Billerica, MA, USA), and then quantified using Quantity One software version 4.4.6 (Bio-Rad Laboratories, Inc., Hercules, CA, USA), normalized to GAPDH.

Statistical analysis. The raw data were analyzed by Origin 8.5 software (OriginLab, Northampton, MA, USA). Results of data analysis were expressed as the means \pm standard error of the mean (SEM) with the number of experiments indicated in the figure legends. Behavioral experiments were statistically analyzed by one-way ANOVA. Tukey's test was employed to compare the differences between all the groups. For all statistical tests, a value of $\mathrm{P}<0.05$ was considered to indicate a statistically significant difference.

\section{Results}

Lithium improves behavioral performance in an MPTPinduced Parkinson mouse model. To examine the effects lithium had on MPTP-induced Parkinson mice, behavioral tasks were performed after 5 weeks of lithium treatment. 

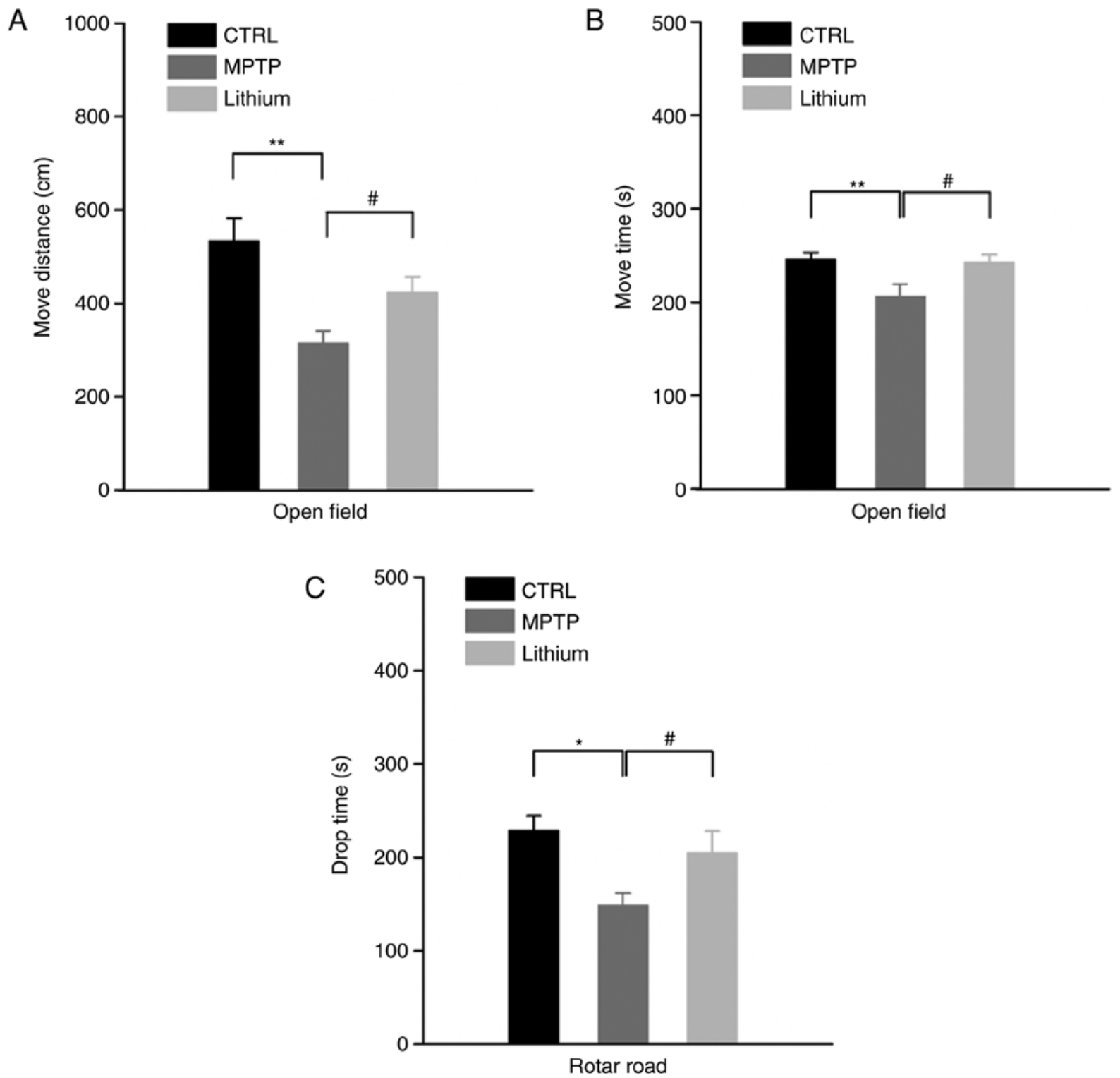

Figure 1. Effects of lithium on behavioral performance of an MPTP-induced PD model. (A and B) Results of behavioral tests revealed that the locomotor activity of MPTP-treated mice was significantly reduced $\left({ }^{*} \mathrm{P}<0.05\right.$ and $\left.{ }^{* *} \mathrm{P}<0.01, \mathrm{n}=10\right)$ and lithium treatment satisfactorily relieved this alteration $\left({ }^{\#} \mathrm{P}<0.05\right.$, $\mathrm{n}=10$ ). (C) In rotarod tests, the compound also prolonged the drop time of MPTP-treated mice ( ${ }^{*} \mathrm{P}<0.05$, MPTP group vs. the control group, $\mathrm{n}=10$; ${ }^{\#} \mathrm{P}<0.05$, lithium group vs. the MPTP group, $\mathrm{n}=10$ ). MPTP, 1-methyl-4-phenyl-1,2,3,6-tetrahydropyridine; PD, Parkinson's disease.

The results revealed a significant reduction in the locomotor activity in the MPTP-treated mice and lithium treatment relieved this effect satisfactorily. As revealed in Fig. 1A and B, the average movement distance was $534.23 \pm 48.01 \mathrm{~cm}$ and the average activity time was $246.33 \pm 6.48 \mathrm{sec}$. Following impairment, the movement distance and activity time values were reduced to $315.09 \pm 25.59 \mathrm{~cm}$ (MPTP group vs. CTRL group, $\mathrm{P}<0.01$ ) and $194.80 \pm 40.79 \mathrm{sec}$ (MPTP group vs. CTRL group, $\mathrm{P}<0.01$ ), respectively, however, after lithium administration for 5 weeks, those values rebounded to $423.40 \pm 33.21$ (lithium group vs. MPTP group, $\mathrm{P}<0.05$ ) $\mathrm{cm}$ and $242.75 \pm 8.05 \mathrm{sec}$ (lithium group vs. MPTP group, $\mathrm{P}<0.05)$, respectively. Furthermore, in rotarod tests, the compound also prolonged the drop time of MPTP-treated mice, resulting in an increase in the drop time for MPTP-treated mice from $148.59 \pm 13.03 \mathrm{sec}$ (MPTP group vs. CTRL group, $\mathrm{P}<0.05$ ) to $204.70 \pm 23.27 \mathrm{sec}$ (lithium group vs. MPTP group, $\mathrm{P}<0.05)$ bringing it close to that of the control group results $228.62 \pm 15.79 \mathrm{sec}$ (lithium group vs. CTRL group, P $>0.05$; Fig. 1C).

Lithium alleviates typical pathological alterations in the substantia nigra region of MPTP-impaired mice. A reduction in TH-positive neurons and an increase in expression of $\alpha$-synuclein are both characteristic of PD pathology. Corresponding pathological changes were also observed in MPTP-impaired mice, and lithium treatment was able to mitigate these alterations as well. Using immunohistochemical staining, a significant loss of $\mathrm{TH}^{+}$neurons in the substantia nigra regions of MPTP-impaired mice at 6 weeks (48.60 $\pm 0.022 \%$ reduction; $\mathrm{P}<0.01$ compared to control) was revealed. Conversely, the number of $\mathrm{TH}^{+}$neurons rebounded considerably after lithium treatment $(32.20 \pm 0.029 \%$ increased; $\mathrm{P}<0.01$ compared to MPTP group; Fig. 2). In addition, the 
A
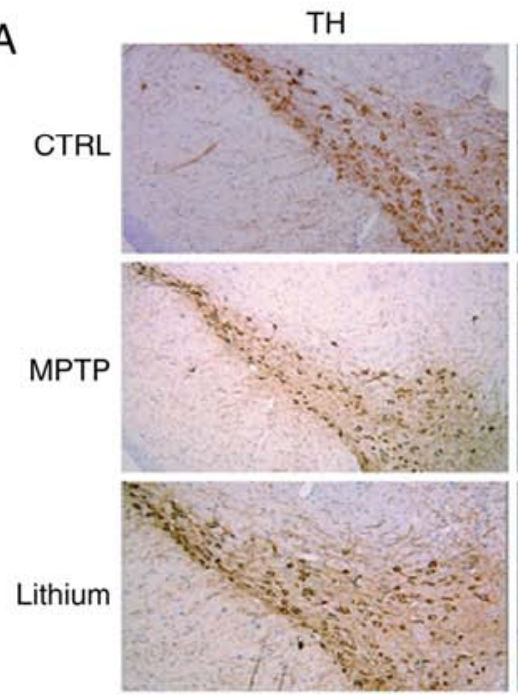
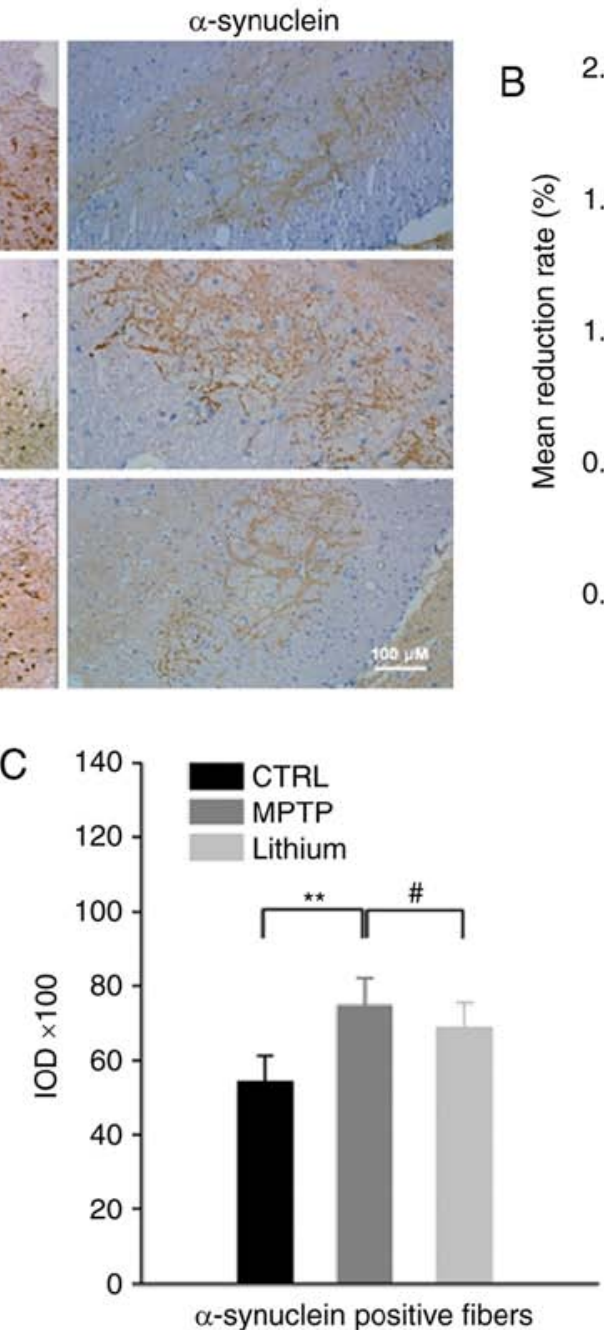

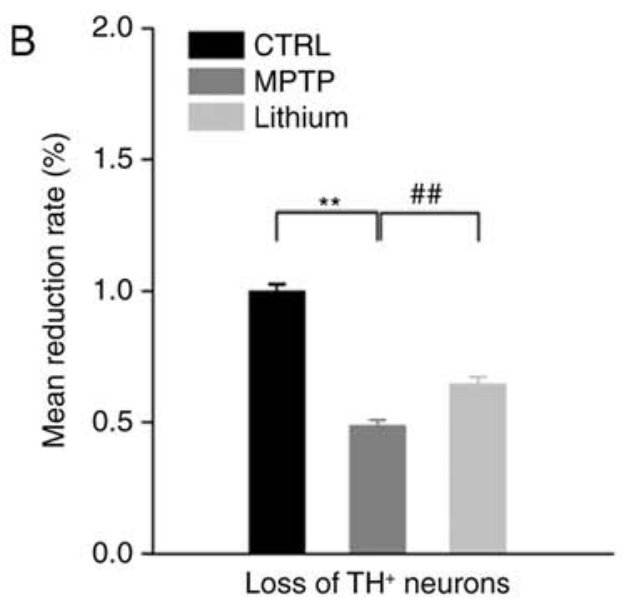

Figure 2. Lithium alleviates typical pathological alterations in the substantia nigra region. (A and $\mathrm{B}$ ) Significant loss of $\mathrm{TH}^{+}$neurons in the substantia nigra region of MPTP-impaired mice at 6 weeks $\left(48.60 \pm 0.022 \%\right.$ reduction; ${ }^{* *} \mathrm{P}<0.01$ compared to control). The number of $\mathrm{TH}^{+}$neurons rebounded considerably (32.20 $\pm 0.029 \%$ increased; ${ }^{\#} \mathrm{P}<0.01$ compared to the MPTP group). (A and C) The integrated IOD of $\alpha$-synuclein-positive fibers in MPTP-impaired mice increased from $54,370 \pm 699$ to $74,790 \pm 736$ (37.5\% increase; ${ }^{* *} \mathrm{P}<0.01$ compared to control), and decreased to $68,958 \pm 654$ after lithium treatment $(7.78 \%$ decrease; ${ }^{\text {P }}<0.05$ compared to the MPTP group). MPTP, 1-methyl-4-phenyl-1,2,3,6-tetrahydropyridine; IOD, optical density value.

integrated optical density value (IOD) of $\alpha$-synuclein-positive fibers in MPTP-impaired mice increased from 54,370 \pm 699 to $74,790 \pm 736$ (37.5\% increased; $P<0.01$ compared to the control), however, after lithium treatment it decreased to $68,958 \pm 654$ (11.3\% increased; $\mathrm{P}<0.05$ compared to the MPTP group; Fig. 2 ).

Lithium increases the methylation of SNCA intron 1 in the substantia nigra of MPTP-impaired mice. To investigate whether epigenetic changes may contribute to the dysregulation of SNCA expression in the MPTP-induced PD animal model, DNA from the substantia nigra regions of each group was analyzed. Significantly fewer methylated $\mathrm{CpG}$ sites in the DNA of PD mice were revealed. There was a significant decrease in the mean methylation rate of SNCA $(-926 /-483$; intron 1) in PD mouse substantia nigra $(2.05 \pm 0.41 \%, \mathrm{P}<0.01)$ when compared with that determined in the control group $(3.62 \pm 0.58 \%)$. Although the methylation rate was lower $(2.38 \pm 0.46 \%)$ in the lithium-treated group when compared to the control group, the opposite was revealed with comparison to the MPTP group, which resulted in a significant increase in the methylation rate $(\mathrm{P}<0.05$; Fig. 3$)$.
Lithium alters the miRNA and protein expression profiles in the substantia nigra of MPTP-impaired mice. The role of miRNAs in the pathogenesis of Parkinsonism has attracted increasing attention. Furthermore, the recent discovery of miRNA expression and regulation has led to the consideration of lithium as a novel mechanism of neuroprotection. SurePrint Mouse miRNA Microarrays (Agilent Technologies, Inc.) were utilized to profile changes in the miRNA expression of MPTP-impaired mice. The results revealed alterations in the expression levels of 39 miRNAs $>1.5$-fold in the substantia nigra of PD mice (data not shown). The results also revealed an upregulation in the expression of miR-148a in PD mice, an effect which was reversed after lithium treatment, resulting in a downregulation of this particular miRNA. RT-qPCR confirmed this result (Fig. 4A). Previous research has indicated that miR-148a could be considered as a potential inhibitor of DNMT1, a DNA methylase, and thus play a role in the establishment and regulation of tissue-specific patterns of methylated cytosine residues. Further RT-qPCR results revealed decreased expression of DNMT1 in the substantia nigra of PD mice compared to the control, and a 

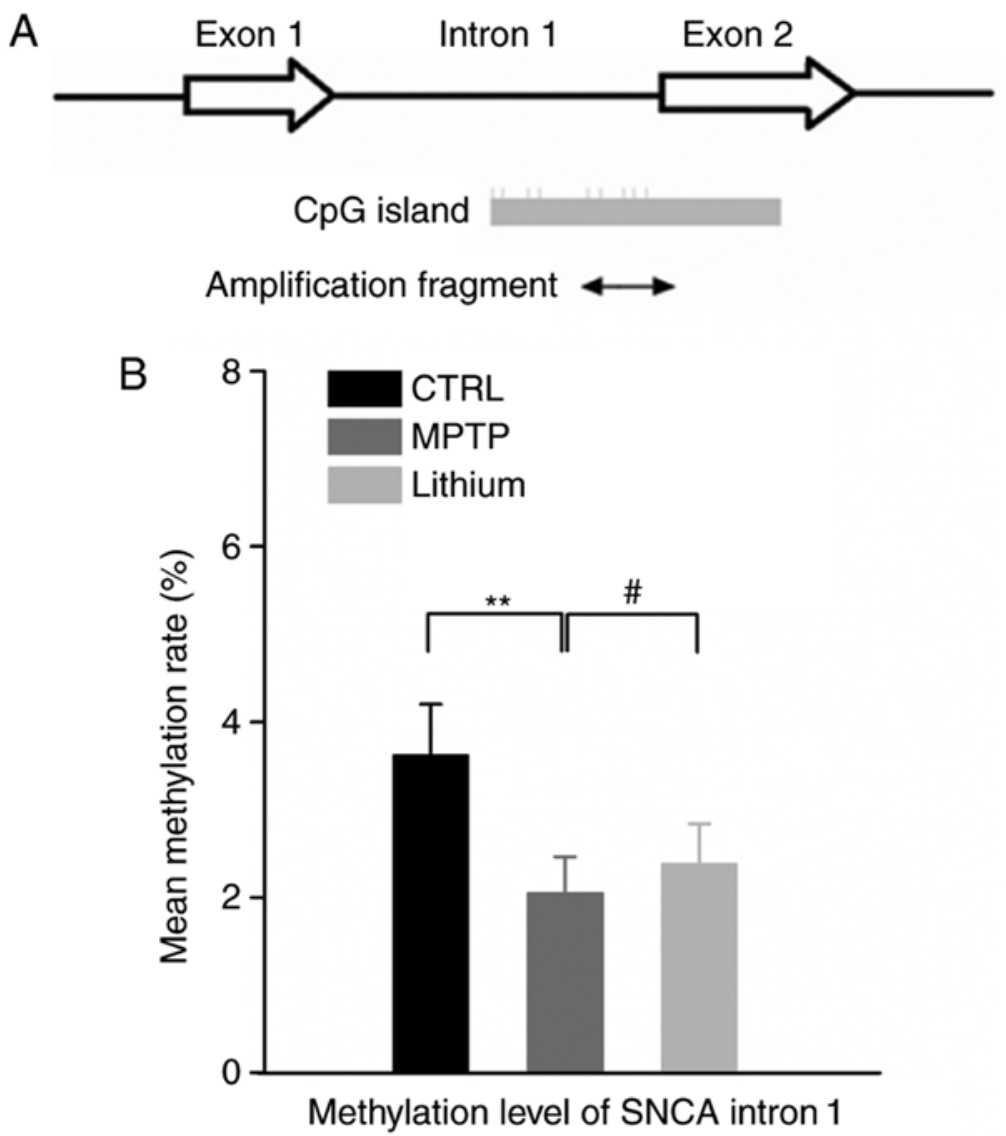

Figure 3. Enhancement of lithium on the methylation of SNCA intron 1 in substantia nigra. (A and B) Bisulfite specific cloning-based sequencing revealed that the mean methylation rate of SNCA $\left(-926 /-483\right.$; intron 1) in PD mouse substantia nigra decreased significantly when compared with control mice $\left({ }^{* *} \mathrm{P}<0.01\right)$ In the lithium-treated group, although the methylation rate remained lower compared to the control group, the opposite was revealed in a comparison with the MPTP group, with a significant increase in the methylation rate ( $\left.{ }^{*} \mathrm{P}<0.05\right)$. SNCA, $\alpha$-synuclein; PD, Parkinson's disease; MPTP, 1-methyl-4-phenyl-1,2,3,6-tetrahydropyridine.

significant difference was recorded when compared with the lithium-treated group (Fig. 4B). These results were also confirmed by western blot analysis, which presented a significant reduction in the expression of DNMT1 protein in PD mice compared with the control group. Furthermore, lithium treatment resulted in a significant increase in DNMT1 expression in PD mice (Fig. 4C).

Aberrant aggregation of $\alpha$-synuclein is considered to be the major neuropathological characteristic of PD, and $\alpha$-synuclein has been detected in insoluble inclusions within Lewy bodies and neurites. In the present study, a significant increase was observed in the expression of $\alpha$-synuclein in the substantia nigra of mice. However, the increase of $\alpha$-synuclein in PD mice could be attenuated through administration of lithium (Fig. 4D).

\section{Discussion}

Lithium mood stabilizers have long been used to treat epilepsy as well as bipolar disorder (BD), a mental illness that causes deficits in cellular plasticity as well as resiliency (8). Recent studies indicate that lithium could also be effective as a treatment for neurodegenerative diseases through diverse mechanisms. For example, pretreatment with lithium resulted in an obvious decrease in the size of quinolinic acid (QA)-induced lesions of striatum and loss of striatal medium-sized neurons in a rat excitotoxic model (16). In tauopathy mouse models, chronic treatment with lithium decreased the phosphorylation of tau as well as neuronal degeneration mediated by GSK-3 (17), findings which were corroborated with the research that lithium could increase neurogenesis in the rat hippocampus (18). An increasing number of studies have illuminated the beneficial effects of lithium on PD. Lithium chloride could promote dopaminergic differentiation of human immortalized RenVm cells (neuronal stem cell) by increasing expression of tyrosine hydroxylase and $\beta$-catenin marker (19). Moreover, low-dose lithium treatment could also prevent motor impairment, as demonstrated by the open field test, pole test, and rearing behavior. In parkin mutant transgenic mice, lithium prevented parkin-induced dopaminergic striatal degeneration, striatal astrogliosis, and microglial activation (20). The present results revealed that lithium treatment significantly counteracted the reduction in the movement distance as well as activity time of MPTP-treated mice in open field tests. Additionally, it prolonged the drop time of MPTP-treated mice in rotarod tests, which may be relative to its neuroprotective effects on nigral neurons. Clinical studies have revealed that low-dose lithium adjunct therapy may reduce off-time in Parkinson's disease.

It is well known that $\alpha$-synuclein is a key component of Lewy bodies found in PD patients $(21,22)$, of which site 

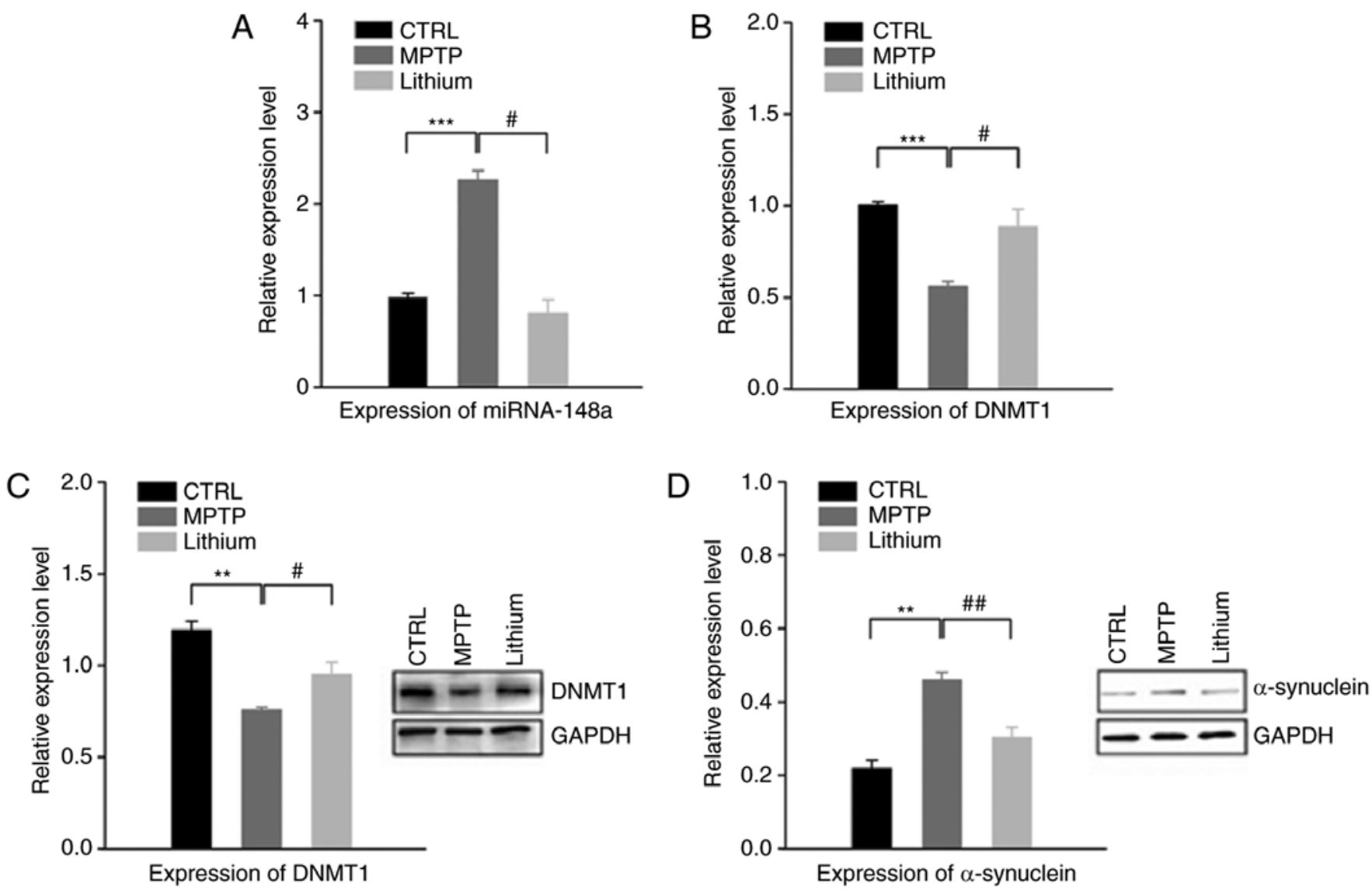

Figure 4. Effects of lithium on the regulation of the miRNA and protein expression profiles in substantia nigra. (A) Results of RT-qPCR assays revealed an upregulation in the expression level of miRNA-148a in the substantia nigra of PD mice compared to that of the control group. However, miRNA-148a expression was downregulated following lithium treatment. (B) The expression level of DNMT1 decreased in the substantia nigra of PD mice compared with the control group, and increased significantly in the lithium-treated group (vs. MPTP group, ${ }^{~} \mathrm{P}<0.05$ ). (C) These results were further confirmed by western blot analysis, which revealed a reduction in DNMT1 in the substantia nigra of PD mice, and a significant difference between the MPTP and lithium group. (D) The expression of $\alpha$-synuclein significantly increased in the substantia nigra of PD mice. This effect was attenuated by oral administration of lithium." $\mathrm{P}<0.01$ and ${ }^{* * * *} \mathrm{P}<0.001$, as indicated; ${ }^{*} \mathrm{P}<0.05$ and ${ }^{\# \#} \mathrm{P}<0.01$, as indicated. PD, Parkinson's disease; MPTP, 1-methyl-4-phenyl-1,2,3,6-tetrahydropyridine.

mutations and multiplications induce familial parkinsonian syndromes with high penetrance (23). Previous studies suggest that the gene SNCA equally harbors significant risk haplotypes for sporadic PD (24) and that MPTP treatment significantly decreases the DNA methylation of SCNA CpGs. In the present study, the effects of chronic lithium treatment were investigated on the neurodegenerative phenotype of an MPTP-induced mouse model of Parkinson's disease. In order to determine whether or not lithium could counteract this alteration, bisulfite specific cloning-based sequencing was performed to analyze the methylation status of SCNA intron 1. Our findings indicated that lithium could effectively prevent the decrease in DNA methylation. We then set out to discriminate the upstream modulator elements of SCNA DNA methylation.

Abundant in all multicellular organisms, miRNAs are 21-24 nucleotides long non-protein-coding RNAs. They are of importance in translational repression as well as mRNA degradation through binding either to the 3'-untranslated regions (3'-UTRs) of mRNAs (25), predominantly, or to coding regions (26). This mechanism allows miRNA-regulating drugs to play a crucial role in regulating the functions of the nervous system. For example, muscarinic M1-receptor knockout mice exhibited mania-like behavioral deficits (e.g., hypersensitivity to amphetamine-induced hyperlocomotion), and lithium treatment normalized these behavioral deficits in part through the enhancement of the M1-receptor-ERK pathway signaling (27). To further investigate whether or not lithium could act effectively by this method in the MPTP-induced PD mouse model, SurePrint Mouse miRNA Microarrays (Agilent Technologies, Inc.) were utilized to profile changes in the miRNA expression of MPTP-impaired mice. It was revealed that, in the substantia nigra of PD mice, 39 miRNAs exhibited altered expression levels at greater than 1.5-fold (data not shown). It was observed that miR-148a was upregulated in PD mice, however lithium treatment could reduce this altered level of expression. DNMT1, a maintenance DNA methylation enzyme, was predicted as a potential target gene of miR-148a, which was revealed to be correlated with DNA hypomethylation as well as $\alpha$-synuclein expression (28). Based on these results, we could postulate that lithium achieved its protective effect against PD-like neurodegenerative symptoms, at least partially, by reducing the expression miR-148a and alleviating the suppression of DNMT1, eventually resulting in a reduction in the generation of neurotoxic $\alpha$-synuclein. Further experiments are required to determine whether or not miR-148a could in fact bind directly to the 3'-UTR of DNMT1 in vivo. 


\section{Acknowledgements}

The authors would like to thank Professor Dazheng $\mathrm{Wu}$ (Putuo Hospital, Shanghai University of Traditional Chinese Medicine) and Associate Professor Peihao Yin (Putuo Hospital, Shanghai University of Traditional Chinese Medicine) for their guidance on the experiments and manuscript.

\section{Funding}

The present study was supported by the National Natural Science Foundation of China (nos. 81202814 and 81603410), the Shanghai Municipal Commission of Health and Family Planning (20124Y116, 20184Y0086), and the Key Speciality Program (no. 2016102A) of Putuo Hospital, Shanghai University of Traditional Chinese Medicine.

\section{Availability of data and materials}

The datasets used during the present study are available from the corresponding author upon reasonable request.

\section{Authors' contributions}

QZ and JT designed the study, performed part of the experiments, interpreted the data and performed the data analysis. JC, YZ, QX, and YB performed part of the experiments. HL, QZ, and JT interpreted the data, drafted the manuscript and revised it critically for intellectual content. All authors read and approved the final version of the manuscript prior to submission.

\section{Ethics approval and consent to participate}

All of the experimental animal protocols complied with the current ethical considerations of Shanghai University of Traditional Chinese Medicine's Animal Ethics Committee, which is in accordance with the National Research Council criteria. All animal experiments and procedures were reviewed and approved by the Institutional Animal Care and Use Committee (IACUC) of Shanghai University of Traditional Chinese Medicine and were performed in accordance with the relevant guidelines and regulations, as well as approved by the guidelines on ethical standards for investigation of experimental pain in conscious animals.

\section{Patient consent for publication}

Not applicable.

\section{Competing interests}

The authors state that they have no competing interests.

\section{References}

1. Tysnes OB and Storstein A: Epidemiology of Parkinson's disease. J Neural Transm (Vienna) 124: 901-905, 2017.

2. Lotankar S, Prabhavalkar KS and Bhatt LK: Biomarkers for parkinson's disease: Recent advancement. Neurosci Bull 33 . 585-597, 2017.
3. Jowaed A, Schmitt I, Kaut O and Wullner U: Methylation regulates alpha-synuclein expression and is decreased in Parkinson's disease patients' brains. J Neurosci 30: 6355-6359, 2010.

4. Matsumoto L, Takuma H, Tamaoka A, Kurisaki H, Date H, Tsuji S and Iwata A: CpG demethylation enhances alphasynuclein expression and affects the pathogenesis of Parkinson's disease. PLoS One 5: e15522, 2010.

5. de Boni L, Tierling S, Roeber S, Walter J, Giese A and Kretzschmar HA: Next-generation sequencing reveals regional differences of the alpha-synuclein methylation state independent of Lewy body disease. Neuromolecular Med 13: 310-320, 2011.

6. Chiu CT, Wang Z, Hunsberger JG and Chuang DM: Therapeutic potential of mood stabilizers lithium and valproic acid: Beyond bipolar disorder. Pharmacol Rev 65: 105-142, 2013.

7. Rao JS, Keleshian VL, Klein S and Rapoport SI: Epigenetic modifications in frontal cortex from Alzheimer's disease and bipolar disorder patients. Transl Psychiatry 2: e132, 2012.

8. Hou L, Xiong N, Liu L, Huang J, Han C, Zhang G, Li J, Xu X, Lin Z and Wang T: Lithium protects dopaminergic cells from rotenone toxicity via autophagy enhancement. BMC Neurosci 16: 82, 2015.

9. Xiong N, Jia M, Chen C, Xiong J, Zhang Z, Huang J, Hou L, Yang H, Cao X, Liang Z, et al: Potential autophagy enhancers attenuate rotenone-induced toxicity in SH-SY5Y. Neuroscience 199: 292-302, 2011.

10. Moors TE, Hoozemans JJ, Ingrassia A, Beccari T, Parnetti L, Chartier-Harlin MC and van de Berg WD: Therapeutic potential of autophagy-enhancing agents in Parkinson's disease. Mol Neurodegener 12: 11, 2017.

11. Fukumoto T, Morinobu S, Okamoto Y, Kagaya A and Yamawaki S: Chronic lithium treatment increases the expression of brain-derived neurotrophic factor in the rat brain. Psychopharmacology (Berl) 158: 100-106, 2001.

12. Emamghoreishi M, Keshavarz M and Nekooeian AA: Acute and chronic effects of lithium on BDNF and GDNF mRNA and protein levels in rat primary neuronal, astroglial and neuroastroglia cultures. Iran J Basic Med Sci 18: 240-246, 2015.

13. Kovacsics CE, Gottesman II and Gould TD: Lithium's antisuicidal efficacy: Elucidation of neurobiological targets using endophenotype strategies. Annu Rev Pharmacol Toxicol 49: 175-198, 2009.

14. Livak KJ and Schmittgen TD: Analysis of relative gene expression data using real-time quantitative PCR and the 2(-Delta Delta C(T)) method. Methods 25: 402-408, 2001.

15. Ribeiro D, Ellwanger K, Glagow D, Theofilopoulos S, Corsini NS, Martin-Villalba A, Niehrs C and Arenas E: Dkk1 regulates ventral midbrain dopaminergic differentiation and morphogenesis. PLoS One 6: e15786, 2011.

16. Senatorov VV, Ren M, Kanai H, Wei $\mathrm{H}$ and Chuang DM: Short-term lithium treatment promotes neuronal survival and proliferation in rat striatum infused with quinolinic acid, an excitotoxic model of Huntington's disease. Mol Psychiatry 9: 371-385, 2004.

17. Noble W, Planel E, Zehr C, Olm V, Meyerson J, Suleman F, Gaynor K, Wang L, LaFrancois J, Feinstein B, et al: Inhibition of glycogen synthase kinase- 3 by lithium correlates with reduced tauopathy and degeneration in vivo. Proc Natl Acad Sci USA 102: 6990-6995, 2005.

18. Chen G, Masana MI and Manji HK: Lithium regulates PKC-mediated intracellular cross-talk and gene expression in the CNS in vivo. Bipolar Disord 2: 217-236, 2000.

19. Soleimani $M$ and Ghasemi N: Lithium chloride can induce differentiation of human immortalized RenVm cells into dopaminergic neurons. Avicenna J Med Biotechnol 9: 176-180, 2017.

20. Lieu CA, Dewey CM, Chinta SJ, Rane A, Rajagopalan S, Batir S, Kim YH and Andersen JK: Lithium prevents parkinsonian behavioral and striatal phenotypes in an aged parkin mutant transgenic mouse model. Brain Res 1591: 111-117, 2014.

21. Spillantini MG, Schmidt ML, Lee VM, Trojanowski JQ, Jakes R and Goedert M: Alpha-synuclein in Lewy bodies. Nature 388: 839-840, 1997.

22. Braak H, Del Tredici K, Rub U, de Vos RA, Jansen Steur EN and Braak E: Staging of brain pathology related to sporadic Parkinson's disease. Neurobiol Aging 24: 197-211, 2003.

23. Singleton AB, Farrer M, Johnson J, Singleton A, Hague S, Kachergus J,Hulihan M,Peuralinna T, Dutra A, Nussbaum R, et al: alpha-Synuclein locus triplication causes Parkinson's disease. Science 302: 841, 2003.

24. Mizuta I, Satake W, Nakabayashi Y, Ito C, Suzuki S, Momose Y, Nagai Y, Oka A, Inoko H, Fukae J, et al: Multiple candidate gene analysis identifies alpha-synuclein as a susceptibility gene for sporadic Parkinson's disease. Hum Mol Genet 15: 1151-1158, 2006. 
25. Lai EC: Micro RNAs are complementary to 3' UTR sequence motifs that mediate negative post-transcriptional regulation. Nat Genet 30: 363-364, 2002.

26. Forman JJ,Legesse-Miller A and Coller HA: A search for conserved sequences in coding regions reveals that the let-7 microRNA targets Dicer within its coding sequence. Proc Natl Acad Sci USA 105: 14879-14884, 2008.
27. Creson TK, Austin DR, Shaltiel G, McCammon J, Wess J, Manji HK and Chen G: Lithium treatment attenuates muscarinic M(1) receptor dysfunction. Bipolar Disord 13: 238-249, 2011.

28. Desplats P, Spencer B, Coffee E, Patel P, Michael S, Patrick C, Adame A, Rockenstein E and Masliah E: Alpha-synuclein sequesters Dnmt 1 from the nucleus: A novel mechanism for epigenetic alterations in Lewy body diseases. J Biol Chem 286: 9031-9037, 2011. 